\title{
A Method of Enhancing Fault Delineation Based on Reflection Strength AC Component Filtering
}

\author{
Chen Zhigang ${ }^{1, ~ *}$, Tian Shuling ${ }^{2}$, Sun Xing ${ }^{1}$, Wang Yuzhu ${ }^{1}$, Han Yuchun ${ }^{1}$, Ma Hui ${ }^{1}$, Chen Jie ${ }^{1}$ \\ ${ }^{1}$ Geological Research Center, Bureau of Geophysical Prospect, Zhuozhou, China \\ ${ }^{2}$ Bureau of Geophysical Prospect International, Zhuozhou, China
}

Email address:

chenzhigang@cnpc.com.cn (Chen Zhigang)

${ }^{*}$ Corresponding author

To cite this article:

Chen Zhigang, Tian Shuling, Sun Xing, Wang Yuzhu, Han Yuchun, Ma Hui, Chen Jie. A Method of Enhancing Fault Delineation Based on Reflection Strength AC Component Filtering. American Journal of Physics and Applications. Vol. 6, No. 4, 2018, pp. 97-103.

doi: 10.11648/j.ajpa.20180604.14

Received: June 26, 2018; Accepted: August 27, 2018; Published: October 12, 2018

\begin{abstract}
Ant tracking technique is a widely used seismic interpretation method of identifying faults in the field of oil and gas exploration and development. However, due to its poor noise immunity, the fault identification effect of ant tracking could be easily affected by the quality of seismic data. Usually, two types of methods can be used to improve the effect of ant tracking, to improve the algorithm of ant tracking or to remove the noise of the seismic data. The first method is usually carried out by the research personnel, and it will take quite a long time before it can be integrated into the software, therefore, the de-noising method is more realistic for the interpreters. This paper puts forward a method of improving the effect of ant tracking by using AC component filtering of reflected intensity. In this method, the structural orientation filtering of the original seismic data is carried out first, and then a coherence cube is calculated based on multiple seismic trace dip scanning. Next, a filtering will be carried out on the coherence cube by using the $\mathrm{AC}$ component of the reflected intensity, and then the positive value after the filtering will be set to zero. Finally, the ant tracking will be processed based on the data volume. The improved ant tracking has a better fault identification effect with a higher fault identification rate, which is more favorable for the detailed interpretation of faults.
\end{abstract}

Keywords: Ant Tracking, Reflection Strength AC Component, Dip Scanning, Coherence Cube, Fault Identification

\section{Introduction}

Geophysicists first proposed the ant algorithm by simulating the behavior of ant foraging in the natural world, and achieved the global optimization through the information transmission between the artificial ant intelligence groups. Essentially, a large number of electronic "ants" are set in the seismic dataset and make the "ant" move forward along the possible fault plane and simultaneously issue the "pheromones". The "ant" that moves forward along the fault can trace the fault plane. If encountering the expected fault plane, it will use the "pheromones" to make an obvious mark. For the plane that is unlikely to be faults, it will not mark or mark only inconspicuously [1]. After Dorigo, Pedersen systematically proposed the ant tracking process [2]. Aqrawi combined edge-preserving smoothing filtering with ant tracking to further improve the ant-tracking process [3]. Ever since Schlumberger integrated it into software, the ant tracking as a low-order fault and fracture identification method has been widely used in oil and gas exploration and development around the world [4-8]. With the widespread application of ant tracking, the disadvantage of poor noise immunity is gradually exposed. In recent years, the researchers have further improved the ant-tracking effect by improving the ant-tracking algorithm or using other seismic attribute dataset instead of coherence cube in combination with the ant tracking. Yan Zhe et al. proposed a direction-constrained ant algorithm to identify the fractures [9]. Lei Xiaozhen et al. proposed a parallel ant colony algorithm [10]. Zhang Jihui proposed an evolutionary ant colony algorithm [11]. Sun et al. combined the frequency division technology with ant tracking [12]. Zhao Wei combined the gray-scale mutants with ant-tracking [13]. 
The effect of ant tracking is improved through above efforts, but there are still two problems. First, the algorithm has a long improvement period and cannot be integrated into mature software in time, so it is not widely used. Second, the replacement of the coherence cube with the seismic attribute dataset cannot address the weakness of poor noise immunity. Reflection strength AC component is a very common seismic attribute, which is mainly used for reservoir prediction. Due to its simple algorithm and early appearance, few literatures have discussed its application. Through calculating the difference between the seismic dataset and the median filter of this attribute in the longitudinal direction, data with more than half of energy and less than half of energy can be separated. In this paper, based on the conventional ant tracking process, the coherence cube is filtered by the reflection strength $\mathrm{AC}$ component to separate the fault data and the non-fault data, so as to achieve the purpose of de-noising, and finally realize the de-noising of ant dataset.

\section{Method and Principle}

\subsection{Reflection Strength AC Component Filtering}

Seismic waves are generally considered to be analytical signals $A(t)$, which is referred to as complex trace in seismic processing. If the real part of seismic trace is $x(t)$, and the imaginary part of seismic trace is $y(t)$, the complex trace can be written as:

$$
A(t)=x(t)+i y(t)
$$

In Formula (1), the imaginary part of seismic trace is an orthogonal trace of the real part, and it can be obtained by Hilbert transform.

The real part of seismic trace $x(t)$ can be considered as a slowly changing cosine vibration:

$$
x(t)=R(t) \cos \theta(t)
$$

In Formula (2), $R(t)$ is the amplitude envelope of vibration; $\theta(t)$ is the phase of vibration.

The imaginary part of seismic trace $y(t)$ can be expressed as:

$$
y(t)=R(t) \sin \theta(t)
$$

The instantaneous amplitude $R(t)$ can be obtained when the real part and imaginary part of seismic traces are known. The instantaneous amplitude is also called the reflection strength or amplitude envelope, that is:

$$
R(t)=|A(t)|=\sqrt{x^{2}(t)+y^{2}(t)}
$$

The reflection strength $\mathrm{AC}$ component is the reflection strength minus the reflection strength DC component, where the DC component is equivalent to the low-frequency component. As shown in Figure. 1a, the blue waveform represents the reflection strength or amplitude envelope of the dataset, the red line represents the trend line or low-frequency component of the dataset, and the low-frequency component is approximately equivalent to the median of the coherent data. Since the reflection strength AC component is the difference between the reflection strength and the low-frequency component, the reflection strength AC component greater than the red median in the blue waveform is a positive value, and the reflection strength $\mathrm{AC}$ component smaller than the red median is a negative value. Since the coherence cube has the low coherent values that represent the fault factors and the high coherent values represent the non-fault noises, after calculating the reflection strength $\mathrm{AC}$ component of the coherence cube, the low coherent values of the faults become negative and the high coherent values of the non-fault interference become positive, where the zero-filling positive values can achieve filtering of coherence cube noise. For many interpreters, it may be thought that the coherent noise of non-fault is filtered by a constant value, but because the coherence cube has different numerical ranges at different depths and positions, so the constant numerical method is only applicable to the local scope and the target layer and is not applicable to the entire coherence cube (Figure 1b). The reflection strength $\mathrm{AC}$ component method is a dynamic numerical filter that varies with depth and plane and is universal for the entire coherence cube.

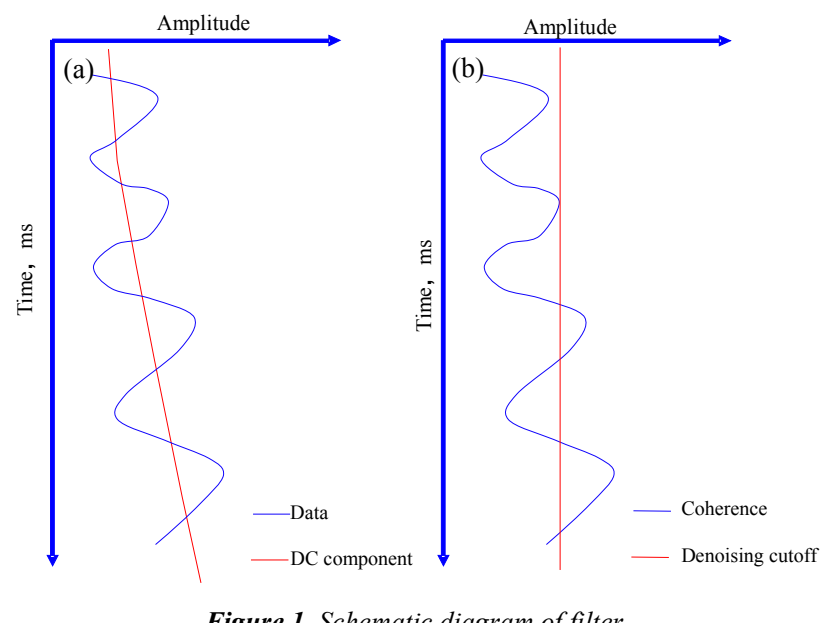

Figure 1. Schematic diagram of filter.

(a) Schematic diagram of RST AC filter; (b) Schematic diagram of constant threshold filter.

\subsection{Improved Ant Tracking Workflow}

The improved ant tracking can be divided into four steps:

(1) Edge preserving smooth filtering. The edge preserving smooth filtering is used to enhance the faults of the original seismic data before the coherent processing, that is, to preserve the fault part and smooth the non-faulted part, so as to achieve the purpose of enhancing the fault. Structure-oriented filtering is a relatively advanced method for edge-preserving smooth filtering. First, the seismic dataset is oriented 
and analyzed, and the dip is calculated along the direction of line and the track. Then edge detection is performed along the direction of the event axis to determine possible reflection termination form, i.e. detect the possible fault locations. Finally the edge preserving and non-edge smooth filtering are carried out along the orientation [14].

(2) Coherent cube operations. The basic dataset of ant tracking is a coherence cube. In order to obtain a better ant tracking effect, a third-generation coherent algorithm based on multi-trace dip scanning should be adopted, i.e. eigenvalue coherence[15,16,17,14].

(3) Reflection strength $\mathrm{AC}$ component filtering. The reflection strength $\mathrm{AC}$ component filtering is the focus of this paper, and it is divided into two steps. First, the reflection strength $\mathrm{AC}$ component is performed on the coherence cube, and the operation process is very simple. Various seismic interpretation software provide this method, where just the coherence cube is input, the line and track range of the operation is selected, and the corresponding time window of dataset operation is provided. The system can automatically calculate without any other parameters. Then, the final filtering is realized when all values of operation result greater than 0 are changed to zero.

(4) Ant tracking for the filtered coherence cube. The most important parameter affecting the effect of ant tracking is the ant step size, where the step size range is $2-10$

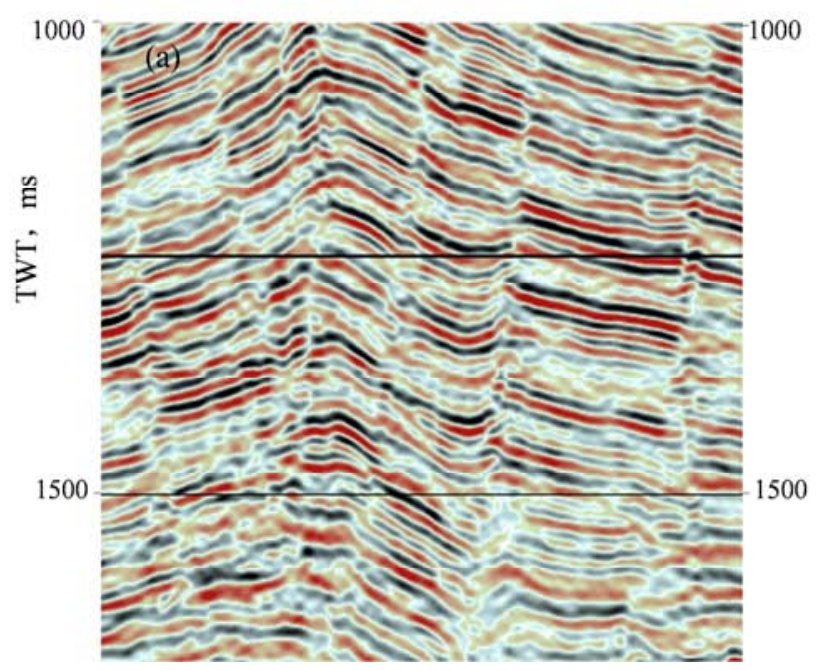

and the default step size is 3 . This parameter defines the ant search step size using the number of samples and determines the single step size of each ant when searching for local maxima. Increasing this value will make each ant search further but reduce the accuracy.

\section{Application}

Taking an oilfield in the east as an example, the process and effect of conventional ant tracking and the ant tracking based on the reflection strength $\mathrm{AC}$ component filtering are introduced.

\subsection{Conventional Ant Tracking}

For the process of conventional ant tracking, edge preserving smooth filtering is first performed on the original seismic data to enhance the breakpoints of the original seismic data. Then the filtered seismic data is processed by coherent cube operation. Finally, the ant tracking of the coherence cube is performed.

(1) Edge preserving smooth filtering. Figure 2 is a relatively advanced edge preserving smooth filtering, namely the seismic profile before and after structure-oriented filtering. Compared with the original seismic profile (Figure 2a), the fault plane is more clearly defined on the seismic profile (Figure $2 b$ ) after the structure-oriented filtering.

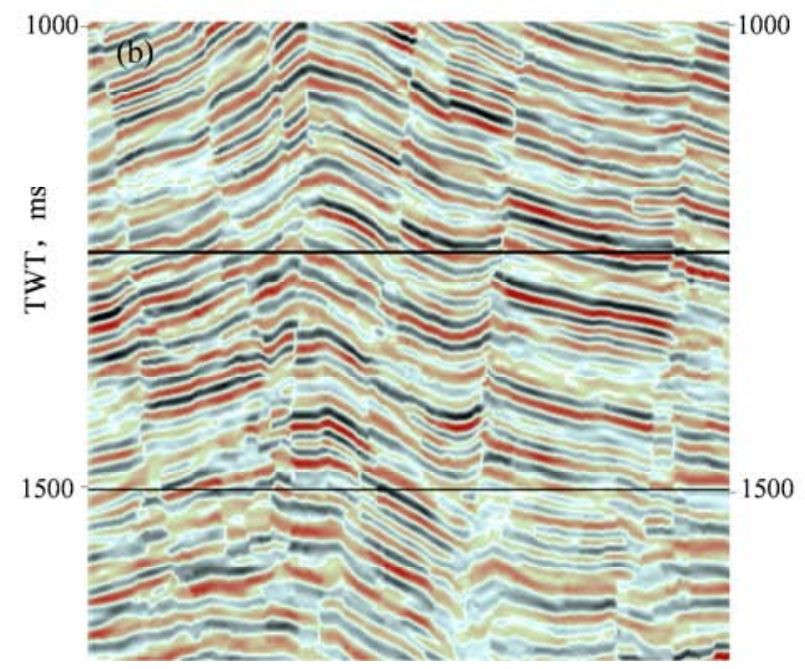

(a) Original seismic section; (b) Structure-oriented filtering section.

Figure 2. Profile comparison before and after structure-oriented filtering.

(2) Coherent cube operation. The coherent cube operation based on multi-trace dip scanning is performed on the structure-oriented filtering seismic data (Figure 3). The faults in coherent profile (Figure 3a) and the coherent time slice (Figure $3 \mathrm{~b}$ ) are clearly visible and the numerical range is below 100. However, when there is too much interference and the numerical range is greater than 110 , it will show a negative effect on fault interpretation, so there is an improved space for the fault identification. 

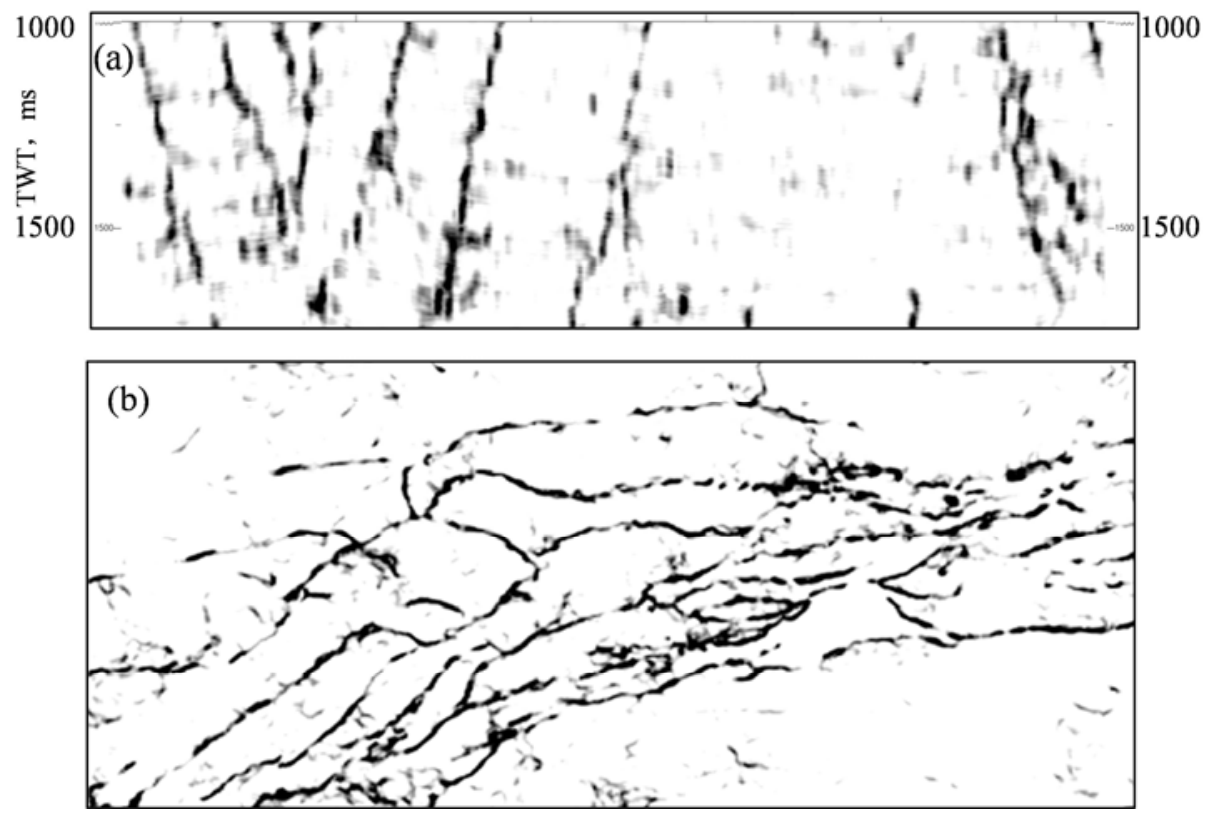

(a) Coherent section; (b) Coherent time slice in $1178 \mathrm{~ms}$.

Figure3. Coherent section and time slice.

(3) Coherent ant tracking. The ant tracking of the coherence cube is shown in Figure 3, where the profile and the plane of ant tracking are too fragmented (Figure 4), indicating that the ant tracking algorithm has poor noise immunity. The interference factors with

a numerical range greater than 110 in the coherent body in Figure 3 are amplified by ant tracking. For interpreters, the conventional ant tracking results are not conducive to fault interpretation.

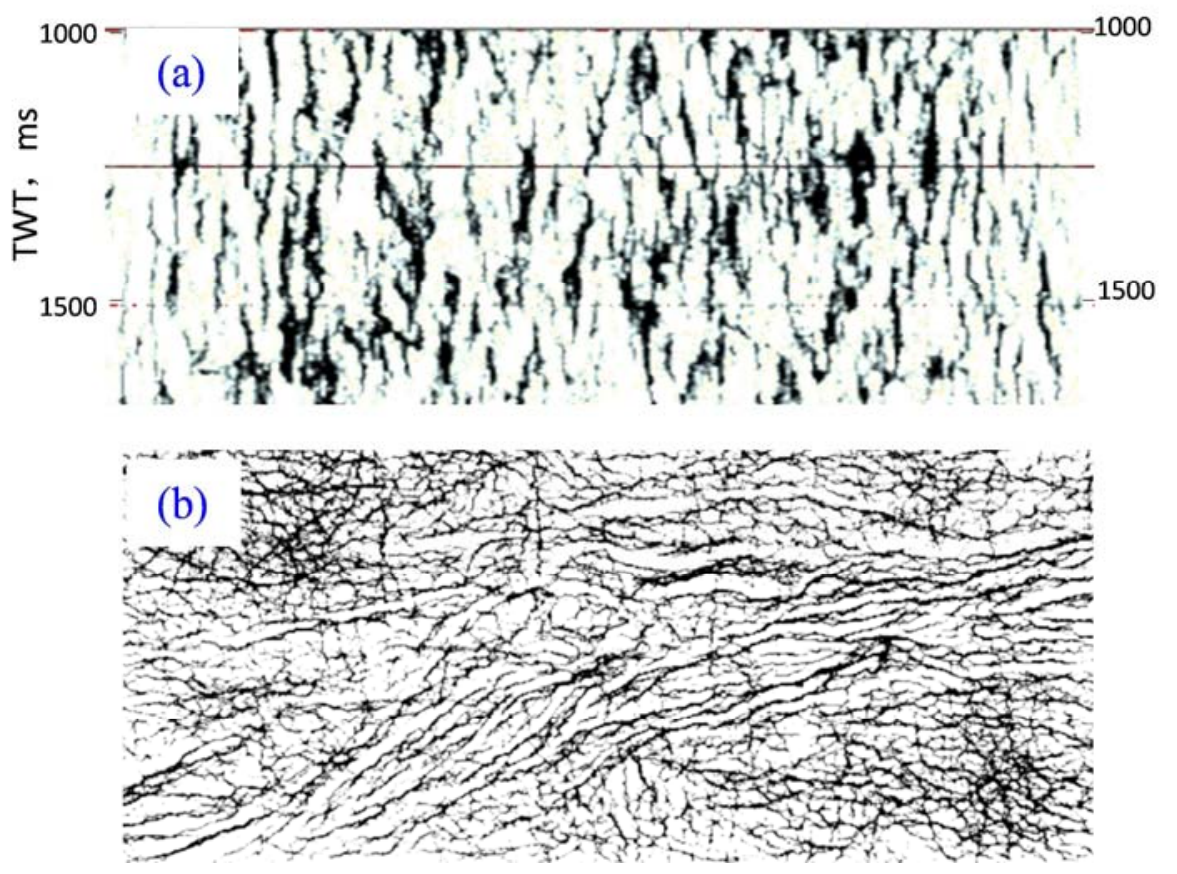

(a) Ant tracking section; (b) Ant tracking time slice in $1178 \mathrm{~ms}$.

Figure 4. Ant tracking section and time slice.

\subsection{Ant Tracking based on Reflection Strength AC Component Filtering}

The ant tracking based on the reflection strength AC component filtering is actually a process that, on the basis of conventional ant tracking, performs ant tracking after the de-noising of the coherence cube. The section and time slice shown in Figure 5 reflect the result of reflection strength AC component filtering of the dataset in Figure 3, and the interference component with a value range of more than 110 in Figure 3 has been basically eliminated in Figure 5 . 

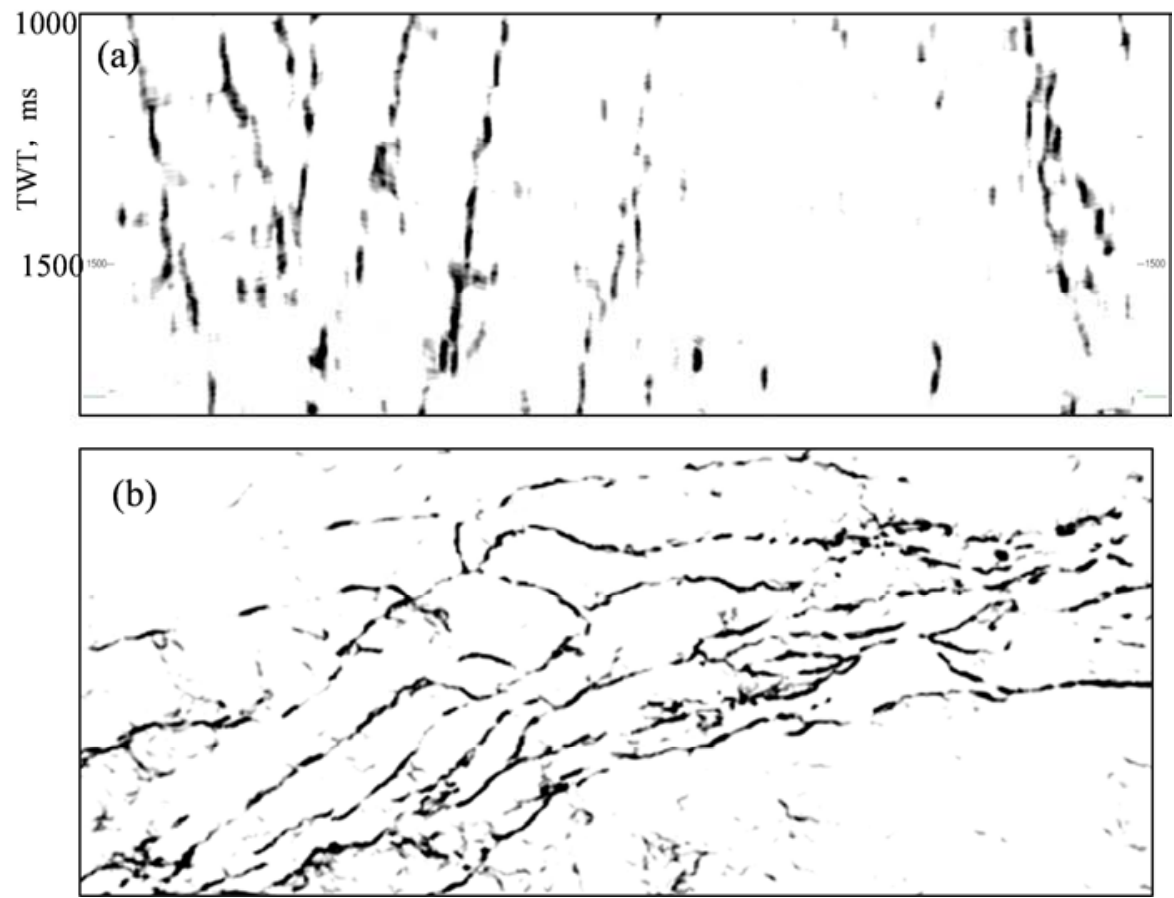

(a) Section of coherent reflection strength AC component filtering; (b) Time slice of coherent reflection strength AC component filtering in $1178 \mathrm{~ms}$.

Figure 5. Section and time slice of coherent reflection strength AC component filtering.

Figure 6 shows the corresponding section and time slice of the dataset of Figure 5 after ant tracking. Compared with the conventional ant tracking (Figure 4), the fault identification effect is obviously improved, where the fault is clearly visible, the combination relationship is clear and it is useful to fault interpretation.
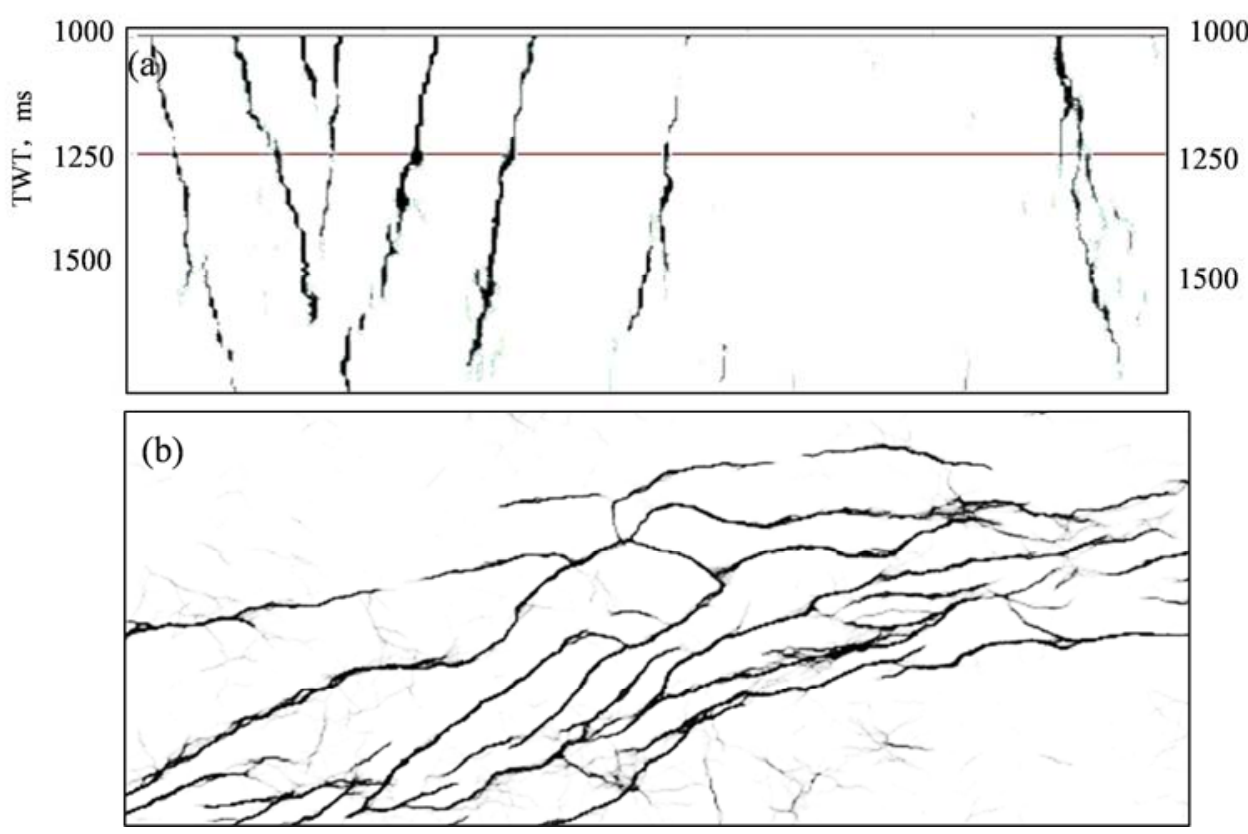

(a) Section of filtered ant tracking; (b) Time slice of filtered ant tracking in $1178 \mathrm{~ms}$.

Figure 6. Section and time slice of filtered ant tracking.

Figure 7 gives a 3D view of the final result, which implies that the improved method by filtering could serve as a 3D fault auto-tracking method. 


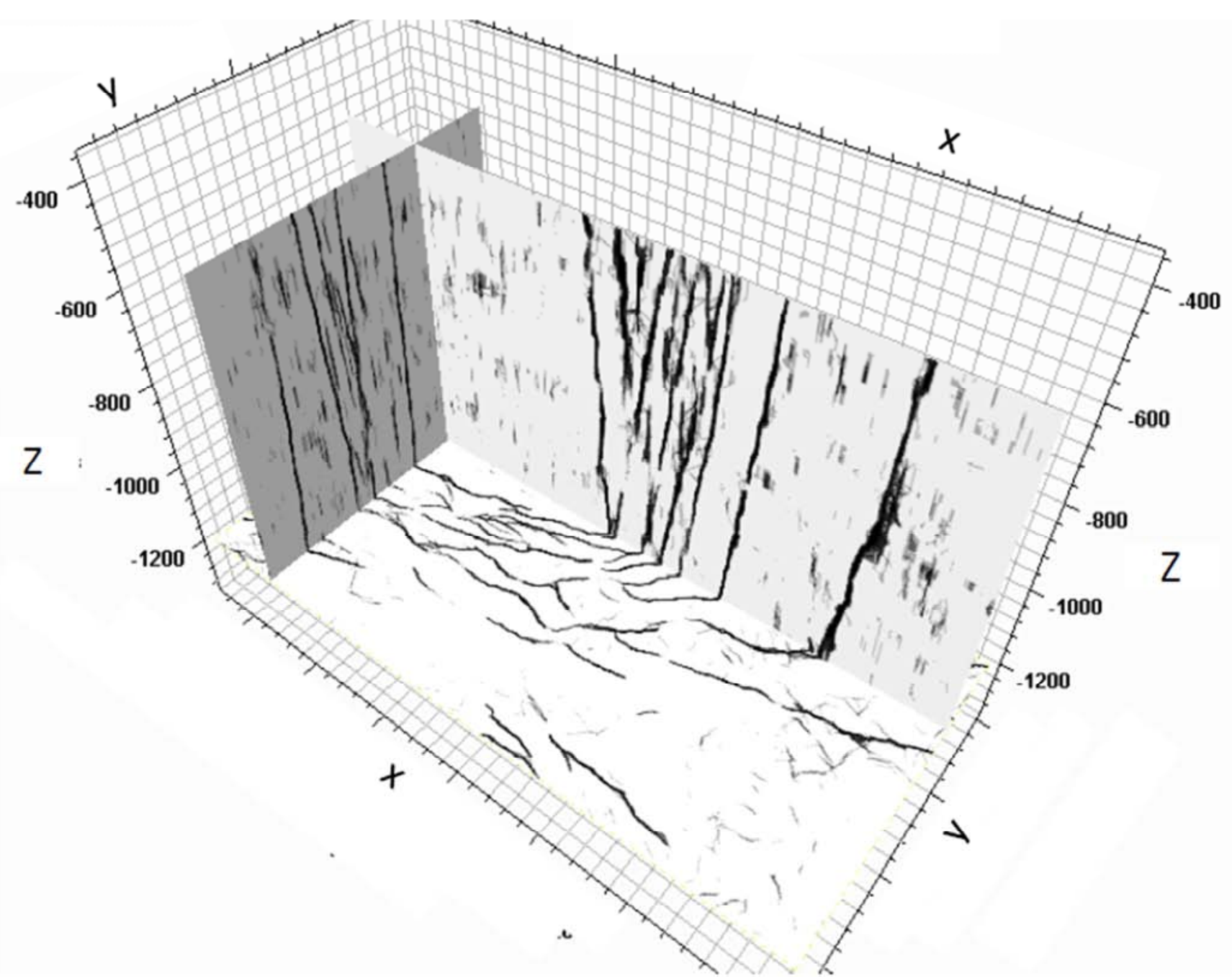

Figure 7. A $3 D$ view of fault mapping result based on the reflection strength AC component filtering.

\section{Conclusions}

(1) Considering the poor noise immunity of conventional ant tracking, a method of de-noising for coherence cube by using the reflection strength AC component filtering is proposed. This method can separate the fault factors and non-fault factors of the coherence cube, and eliminate the non-fault factors. On this basis, the ant tracking technique can avoid the problem of fragmentation of conventional ant-tracking due to poor noise immunity.

(2) The improved ant tracking method has been used to an oilfield in the eastern china, and this new method has been proved to be more effective than the conventional ant tracking method. The processing results can clearly identify the faults and have a higher reference value for seismic interpreters.

\section{Acknowledgements}

The author want to extend their thanks to National Major Science and Technology Project (2016ZX05029005) for it's support.

\section{References}

[1] Dorigo M, Maniezzo V and Colorni A. Ant system: Optimization by a colony of cooperating agent [J]. IEEE Trans on SMC, 1996, 26(1): 1-13.

[2] Pedersen S I, Skov T. Automatic fault extraction using artificial ants [J]. SEG Technical Program Expanded Abstracts, 2002, 21: 512-515.

[3] Aqrawi A. Improved fault segmentation using a dip guided and modified 3D Sobel filter [J]. SEG Technical Program Expanded Abstracts, 2011, 30: 999-1003.

[4] GUO Q, LI R M. Application of ant-tracking technique in Cretaceous structure in Yanmuxi Oilfield [J]. Tuha Oil \& Gas, 2008, 13(1), 39-41.

[5] ZHANG X. Application of ant tracing algorithm in fault automatic interpretation: a case study on Fangheting structure in Pinghu Oilfield [J]. OGP, 2010, 45(2): 278-281.

[6] JIANG X F, LI X Y, LI X M. Application of ant tracking technology in small fault identification [J]. Tuha Oil \& Gas, 2012,17 (4): 323-325. 
[7] LIN C H, GUO L Q, ZHANG W. Fine interpretation of mine geological structure through Ant tracking technology [J]. Coal Geology of China, 2013, 25 (4): 55-59.

[8] ZHOU W, YIn T J, ZHANG Y C. Application of ant tracking technology to fracture prediction: A case study from Xiagou Formation in Qingxi Oilfield [J]. Lithologic Oil\&Gas Reservoir, 2015, 27(6): 111-118.

[9] YAN Z, GU H M. Fault identification by orientation constraint ant colony algorithm [J]. OGO, 2011, 46(4): 614-620.

[10] LEI X Z, LAI W Q. An improved parallel ant colony optimization algorithm [J]. Journal of Gansu Lianhe University (Natural Sciences) 2009, 23 (1): 67.

[11] ZHANG J H, XU X H. A new evolutionary algorithm-Ant colony algorithm $[\mathrm{J}]$. Systems Engineering-Theory \& Practice, 1999, (3): 84-87.

[12] Sun D S and Ling Y. Application of spectral decomposition and ant tracking to fractured carbonate reservoirs [J]. EAGE extended Abstracts, 2011, B035: 23-26.
[13] ZHAO W. A study of 3D seismic fault identification based ant colony Algorithm [D]. Nanjing: Nanjing University of Science and Technology, 2009.

[14] Haskell N.L, Nissen S.E., Lopez J.A, et al., 3-Dseismic coherency and imaging of sedimentological features $[\mathrm{J}]$. Expanded abstracts of the 65th annual internet SEG meeting, 1995, 1532-1534.

[15] Bahorich M.S., Lopez J.A., Haskell N L, et al. Stratigraphic and structural interpretation with 3-D coherence[J]. Expanded abstracts of the 65th annual internet SEG meeting, 1995, 97-100.

[16] Gersztenkorn, A., and K. J. Marfurt, 1996, Eigen structure based coherence computations $[\mathrm{J}]$, 66th Annual International Meeting, SEG, Expanded Abstracts, 328-331.

[17] Gijs C Fehmers and Christian F W Hocker. Fast structural interpretation with structure-oriented filtering [J]. Geophysics, 2003, 68(4): 1286-1293. 